\title{
Improved Association and Disassociation Scheme for Enhanced WLAN Handover and VHO
}

\author{
Sungjin Shin, Donghyuk Han, Hyoungjun Cho, and Jong-Moon Chung \\ School of Electrical and Electronic Engineering, Yonsei University, Seoul 03722, Republic of Korea \\ Correspondence should be addressed to Jong-Moon Chung; jmc@yonsei.ac.kr
}

Received 4 January 2016; Accepted 13 April 2016

Academic Editor: Stavros Kotsopoulos

Copyright (C) 2016 Sungjin Shin et al. This is an open access article distributed under the Creative Commons Attribution License, which permits unrestricted use, distribution, and reproduction in any medium, provided the original work is properly cited.

The number of mobile devices and wireless connections is significantly increasing. Among many wireless protocol types, wireless local area networks (WLANs) are expected to support a significant number of devices. Due to this reason, effective and efficient handover $(\mathrm{HO})$ and vertical handover $(\mathrm{VHO})$ support for WLAN mobile devices is important. A significant improvement in quality of service (QoS) can be obtained by reducing the association and disassociation interruption time for user equipment (UE) servicing real-time applications during WLAN HO and VHO operations. Based on this focus, this paper investigates the problem of using only the received signal strength indicator (RSSI) in HO and VHO decisions, which is what the current IEEE 802.11 based WLAN systems use. Experimental results presented in this paper demonstrate that only using the RSSI results in significant interruption time during $\mathrm{HO}$ to another WLAN access point (AP) or to a cellular base station during VHO. Therefore, in this paper, an improved association and disassociation scheme that can reduce the data interruption time (DIT) and improve the throughput performance is proposed.

\section{Introduction}

Wireless local area networks (WLANs) devices use the received signal strength indicator (RSSI) for association and disassociation [1]. RSSI is the sum of signal power of the serving access point (AP) and the surrounding interference and noise. In an area with the Bluetooth, ZigBee, and other APs using the same frequency band, the level of interference increases and the RSSI level will increase, but the packet success rate (PSR) will degrade, since the PSR depends on the signal to interference and noise ratio (SINR). Therefore, handover (HO) and vertical HO (VHO) based on the RSSI threshold cannot perform properly in places with severe interference. In [2], the HO RSSI threshold is calculated considering the speed and direction of the user equipment (UE). In [3], the session setup as well as the speed and direction of the UE is considered in the calculation of the $\mathrm{HO}$ RSSI threshold. However, these two schemes were designed under the assumption that there will be no other interfering device (e.g., Bluetooth, ZigBee, or other Wi-Fi APs) near the UE. Therefore, under densely located APs and interfering devices, the channel status estimations will be erroneous leading to problematic HO RSSI threshold level settings. In addition, in [4], the outage probability in various levels of RSSI is considered. However, [4] does not provide any HO scheme or interference mitigation/suppression scheme that uses the estimated outage information. Data interruption time (DIT) is defined as the time from the last received data packet before $\mathrm{HO}$ (at the UE) to the time that a packet is received (after HO) through the newly attached AP (or mobile communication base station (BS)). In addition, the partial packet success (PPS) region is defined as a region where the beacon, request to send (RTS), and clear to send (CTS) packet communication are successful but data packet communication consistently fails. In this paper, an improved WLAN association and disassociation scheme that can reduce the DIT and improve the throughput performance during $\mathrm{HO}$ is proposed. The proposed scheme uses PPS detection, and in this region efficient $\mathrm{HO} / \mathrm{VHO}$ is conducted.

\section{Problem Description}

Interference is among the main causes of incorrect decision in $\mathrm{HO}$ techniques that are based on the beacon signal's RSSI. 


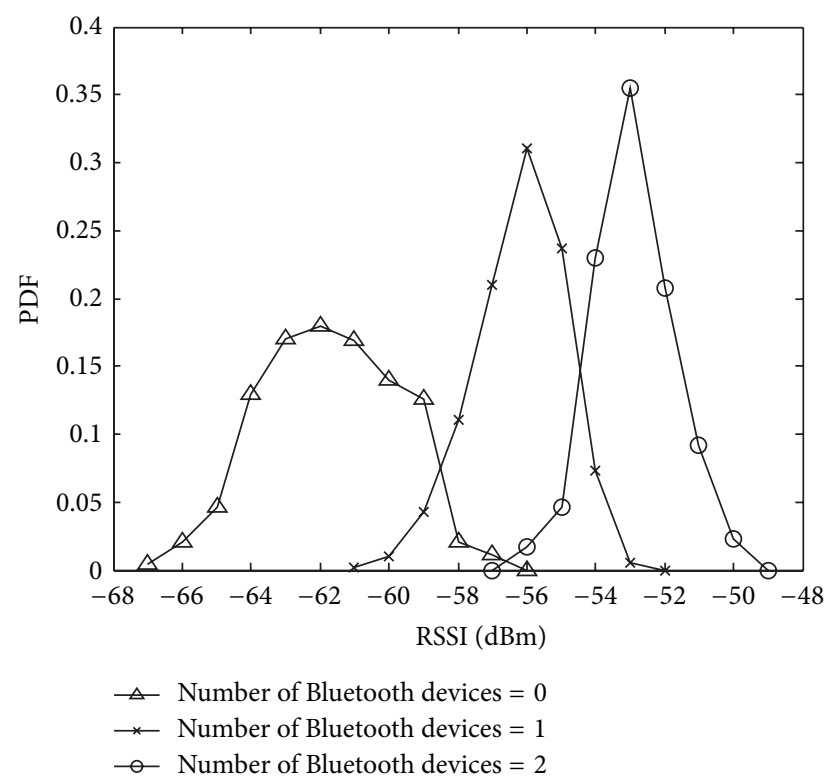

FIGURE 1: RSSI PDF of received beacon packets from AP with varying number of Bluetooth devices.

To illustrate this point, experiments were conducted in real environments, where Figure 1 shows the influence on RSSI measurements based on increasing interference. The interference added to the RSSI of the WLAN device was generated by Bluetooth devices using audio streaming. This experiment was conducted because it is a very common scenario, as it occurs to Wi-Fi users, where a nearby wireless Bluetooth headset may be receiving streaming music from a smartphone. Bluetooth was selected as the interference source to WLAN as it uses the same frequency band as Wi-Fi and also it is one of the most common interference sources to $\mathrm{Wi}-\mathrm{Fi}$ users. For the interference experiment, a Samsung Galaxy S4 (SHV-E330S) smartphone and a Bluetooth headset that uses Bluetooth version 2.1+ enhanced data rate (EDR), Advanced Audio Distribution Profile 1.2 (A2DP), Audio/Video Remote Control Profile 1.4 (AVRCP), and subband coding (SBC) codec were applied. The smartphone and the Bluetooth headset were at a $50 \mathrm{~cm}$ distance and the bit rate of the audio streaming data rate was $372 \mathrm{kbps}$. In the measurements of Figure 1, 10,000 beacon packets were used to compute the RSSI PDF.

In Figure 2, the RSSI measurement results show the difference in packet reception ranges based on different packet types in the PPS region, where measurements were taken at City Hall of Seoul in South Korea. Applications used in the measurements were File Transfer Protocol (FTP) and YouTube. Figure 2 shows the average RSSI of packets based on the distant from the AP, and $N_{B}$ represents the number of APs that were broadcasting beacon packets. " $N_{B}=5$ " represents the lowest interference location and " $N_{B}=94$ " represents the location with the highest level of interference. In the measurements of Figure 2, 150 repetitions were performed to compute the average RSSI. In addition, 200,000 RTS/CTS packets, 200,000 data packets, and 1,800 beacon packets were used. As can be observed in Figure 2, RSSI outage occurs

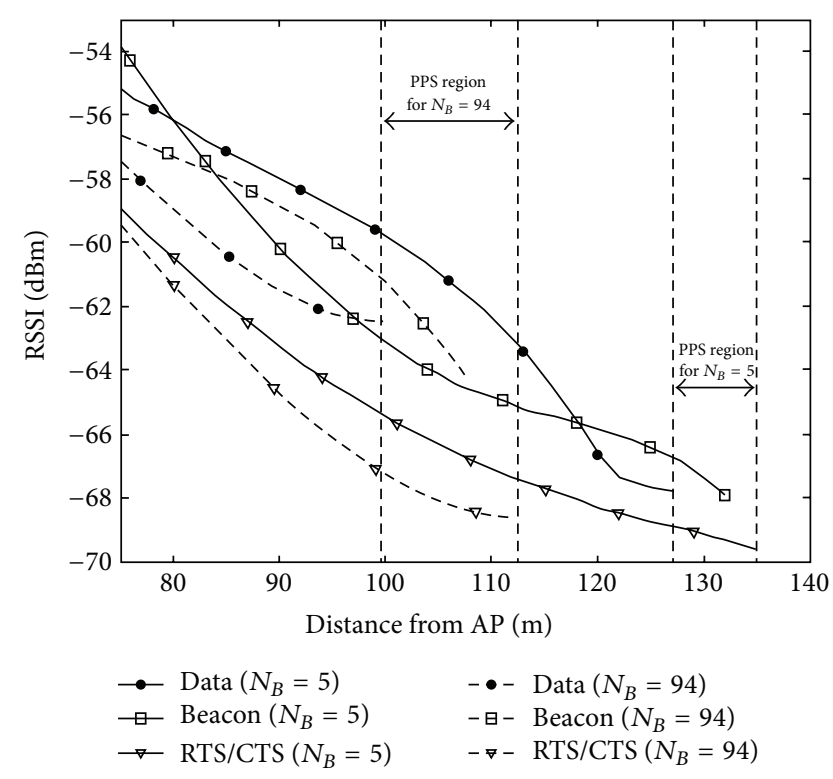

FIGURE 2: RSSI of received RTS/CTS, beacon, and data packets from AP.

at significantly different levels for the RTS (44 bytes), CTS (30 bytes), beacon ( 300 bytes), and data (1,500 bytes) packets. The outage RSSI was $-67.74 \mathrm{dBm}$ in the location with the lowest interference $\left(N_{B}=5\right)$, whereas the outage RSSI was $-62.57 \mathrm{dBm}$ at the highest interference $\left(N_{B}=94\right)$ location.

In order to clarify the relation between the PER and PPS range, hardware and firmware testing was conducted using the kernel program from http://www.multipath-tcp.org/ based on a Linux Ubuntu platform on a Samsung ATIV TAB 7 laptop. For the experiment, the Ubuntu 14.04.3 LongTerm Support (LTS) and kernel v0.90 were installed. In the experiment, the iwlwifi driver (\drivers \net\wireless \iwlwifi) was used and the data rate adaptation algorithm was implemented in the rs.c file (\drivers \net\wireless \iwlwifi \dvm). From the rs.c file, the packet success ratio was obtained, which was computed based on 13,500 packets. The packet success ratio was stored in "window $\rightarrow$ success_ratio". The value of the success ratio and the transmission rate index was obtained using the printk command. From the test using the printk command, the minimum PER was 0.5988, the maximum PER was 0.8814 , and the average PER was 0.7630 when outage of the data packets started to occur. In the PER range of between 0.5988 and 0.8814 , data packet transmission consistently failed but RTS/CTS packets were successfully exchanged, which is why it is defined as the PPS region.

In Figure 2, it can be observed that there is a difference in RSSI level based on the difference in channel environments. When the UE is in the AP's PPS region, it does not only influence these devices, but this may result in an outage of the neighboring area. The reason for this is because the RTS and CTS messages exchanged by the UE and AP (in the PPS region) will block other devices from attempting communication because once the neighboring devices receive a RTS or CTS, these devices will set their network allocation vector (NAV) duration and will not transmit during this 
period to avoid packet collision. But since the pair of devices in the PPS region will reattempt another transition (due to failure of data packet transfer), the pair of devices in the PPS region will resend RTS and CTS messages, which will reset the NAVs of the neighboring devices again. This repeated pattern will result in an outage of the neighboring area of the pair of devices in the PPS region, and, therefore, the neighboring devices will experience extreme difficulty in channel access. To avoid these network performance degrading issues, when a UE is in the PPS region of its AP, the proposed scheme enables the UE to quickly find an alternative AP (or BS) with satisfying quality to establish a connection through association procedures, while the UE also executes disassociation procedures with the former AP that has poor conditions.

\section{Proposed Scheme}

In decreasing signal power or increasing surrounding interference situations, small size packet transmissions may succeed, but large size packet transmissions will fail more frequently. In Figure 2, when the packet size increases, it can be seen that the difference in packet error rate (PER) is significant. Using the characteristic of PSR based on the packet size, a PPS criterion is defined to determine whether the WLAN channel situation is suitable or not for data packet transmission. The PPS criterion for WLAN association and disassociation is defined using the indicator $I_{\mathrm{PPS}}$ :

$$
I_{\mathrm{PPS}}= \begin{cases}1 & I_{\mathrm{RTS} / \mathrm{CTS}}=1, I_{\text {data }}=0 \\ 0 & I_{\mathrm{RTS} / \mathrm{CTS}}=1, I_{\text {data }}=1,\end{cases}
$$

where $I_{\mathrm{RTS} / \mathrm{CTS}}=1$ and $I_{\text {data }}=1$ represent success of a RTS/CTS and data packets, respectively, and $I_{\text {data }}=0$ refers to data packet communication failure. Consequently, $I_{\mathrm{PPS}}=1$ means that the channel environment of the WLAN is not suitable for data packet transmission even though RTS and CTS packets are successfully exchanged.

When using only the PPS criterion for association and disassociation, frequent $\mathrm{HO}$ triggering can occur due to temporary channel errors despite the device being located near the AP. In order to prevent this, the proposed scheme uses SINR as an additional criterion for HO. The SINR criterion uses a minimum SINR threshold, which is based on the data rate used for the application. Above this threshold, even if the RTS/CTS packet transmission succeeds and the data packet transmission fails, the smartphone will continue to use the same WLAN AP because it considers that this packet transmission failure was caused by a temporary channel error. To derive the $\mathrm{HO}$ triggering point, the channel capacity formula $R=W_{\mathrm{AP}} \log _{2}\left(1+\gamma / \Gamma_{\mathrm{AP}}\right)$ is used [5], where $R$ is the maximum data rate, $\gamma$ is the SINR, $W_{\mathrm{AP}}$ is the carrier bandwidth of the WLAN, and $\Gamma_{\mathrm{AP}}$ is channel coding loss factor of the WLAN. The minimum SINR threshold $\gamma_{\min }$ for a $\mathrm{HO}$ modem data rate, $R_{\mathrm{HO}}$, is represented in

$$
\gamma_{\min }=\Gamma_{\mathrm{AP}}\left(2^{R_{\mathrm{HO}} / W_{\mathrm{AP}}}-1\right) .
$$

Using (2), the SINR criterion for WLAN HO is used in the SINR threshold indicator below:

$$
I_{\text {SINR }}= \begin{cases}1 & \gamma \leqq \gamma_{\min } \\ 0 & \gamma>\gamma_{\min } .\end{cases}
$$

When $I_{\text {SINR }}=1$, the SINR of the WLAN AP is clearly insufficient to satisfy the target data rate used by the application. Using (1) and (3), the proposed scheme (PPS and SINR (PS)) for WLAN disassociation and association defines the indicators as below:

$$
\begin{aligned}
& I_{\mathrm{PS}}^{\text {Disasso }}= \begin{cases}1 & I_{\mathrm{SINR}}^{\mathrm{ServAP}}=1, I_{\mathrm{PPS}}^{\mathrm{ServAP}}=1 \\
0 & \text { otherwise, }\end{cases} \\
& I_{\mathrm{PS}}^{\text {Asso }} \\
& \quad= \begin{cases}1 & \left(I_{\mathrm{SINR}}^{\text {TargAP }}=0, I_{\mathrm{PPS}}^{\text {TargAP }}=0\right) \text { or }\left(I_{\mathrm{SINR}}^{\text {TargBS }}=0\right) \\
0 & \text { otherwise. }\end{cases}
\end{aligned}
$$

$I_{\text {SINR }}^{\text {ServAP }}$ and $I_{\mathrm{PPS}}^{\text {ServAP }}$ represent SINR and PPS indicator of the currently serving AP, respectively; $I_{\mathrm{SINR}}^{\text {TargAP }}$ and $I_{\mathrm{PPS}}^{\text {TargAP }}$ represent the SINR and PPS indicator of the target AP, respectively. For the case of $\mathrm{HO}$ to a $\mathrm{BS}, I_{\mathrm{SINR}}^{\text {TargB }}$ represents the SINR of the target BS. When $I_{\mathrm{PS}}^{\text {Disasso }}=1$, WLAN disassociation is executed and when $I_{\mathrm{PS}}^{\text {Asso }}=1$, WLAN association is executed.

The association procedure is first based on satisfaction of the SINR level (which depends on the SINR measurement of the AP's beacon packets). If the SINR level is above $\gamma_{\min }$ (i.e., the minimum SINR threshold), then the next procedure is to check the Probe Request packet. The Probe Request packet is padded to be the same size of an average data packet. The amount of padded bits per Probe Request packet can be represented as $L_{\text {pad }}=L_{\text {data }}-L_{\text {Probe.Rq, }}$, where $L_{\text {data }}$ is the average payload length and $L_{\text {Probe.Rq }}$ is frame body length of the Probe Request. Since each association requires a Probe Request packet to be sent and that packet will be padded to make the packet equivalent to an average data packet size, the overhead that will be added to the wireless network due to the padding bits can be expressed as $L_{\text {pad }} \times R_{\text {Asso }}=$ $\left(L_{\text {data }}-L_{\text {Probe.Rq }}\right) \times R_{\text {Asso }}$, where $R_{\text {Asso }}$ is the average rate of associations conducted per second in the WLAN system. Therefore, transfer success/failure of this packet will indicate potential success/failure of data packets over the alternative (target) AP. If the padded Probe Request packet transfer is successful, then the UE will complete the association procedures to the target AP. The time between two probes to identify the value of the PPS of an alternative (target) AP is $102.4 \mathrm{~ms}$ because this is the time between two beacon packets, which is the starting point of the SINR level measurements in the association procedures. The disassociation procedure is based on the SINR level of the received packets and the PPS phenomena detection, which depends on the RTS/CTS and data packets transfer success/failure conditions. The presented PS scheme can be set up in the form of the pseudocode presented in Algorithm 1. 
(1) Get information of RTS/CTS and data packet transmission success

(2) Compute $\gamma_{\min }$ based on the modem data rate used for the application

(3) If $I_{\mathrm{SINR}}^{\text {ServAP }}=1$ and $I_{\mathrm{PPS}}^{\text {ServAP }}=1$ then

(4) if there are other APs with $\gamma>\gamma_{\min }$ then

(5) select the AP with the largest SINR

(6) if $I_{\mathrm{PPS}}^{\mathrm{TargAP}}=0$ then

(7) execute $\mathrm{HO}$ from serving AP to target $\mathrm{AP}$

(8) elseif there are other BSs with $\gamma>\gamma_{\min }$ then

(9) select the BS with the largest SINR

(10) execute HO from serving AP to target BS

(11) Else remain connected to the current serving AP

Algorithm 1: PS algorithm.

\section{Performance Evaluation}

In the PS scheme, the SINR criterion and the PPS criterion both need to be satisfied for $\mathrm{HO}$ execution. In the following, the performance model of $[6,7]$ was used to represent the DIT and throughput of the proposed PS scheme as $\tau=(1+$ $\left.\left((1-p) /\left(1-p^{m+1}\right)\right) \sum_{i=0}^{m}\left(\left(p^{i}\left(2^{i} \mathrm{CW}-1\right)-(1-p)\right) / 2\right)\right)^{-1}$, $p=1-(1-\tau)^{n-1}\left(1-P_{\mathrm{PE}}^{\mathrm{RTS}}\right)$, where $\tau$ is the probability that a UE transmits a RTS packet in a randomly chosen slot time, $p$ is the probability that a transmitted RTS packet encounters a collision, $m$ is the number of backoff stages, CW is the contention window size, $i$ is the backoff stage (where $i=1, \ldots, m$ ), $n$ is number of contending stations (which are assumed to always have a RTS or data packet ready for transmission), and $P_{\mathrm{PE}}^{\mathrm{RTS}}$ is the RTS packet's PER. The PER of a RTS packet was included in the channel error term, in order to accurately model $p$. The probability of events that can happen in a randomly chosen slot time are derived as $P_{\mathrm{tr}}=1-$ $(1-\tau)^{n}, P_{S}=n \tau(1-\tau)^{n-1}\left(1-P_{\mathrm{PE}}^{\mathrm{RTS}}\right) /\left(1-(1-\tau)^{n}\right), P_{\mathrm{C}}=1-n \tau(1-$ $\tau)^{n-1} /\left(1-(1-\tau)^{n}\right)$, and $P_{E}=n \tau(1-\tau)^{n-1} /\left(1-(1-\tau)^{n} P_{\mathrm{PE}}^{\mathrm{RTS}}\right)$, where $P_{\operatorname{tr}}$ is the conditional probability that at least one transmission occurs in a randomly chosen slot time, $P_{S}$ is the conditional probability that this transmission is successful, $P_{C}$ is the probability that an occurring transmission collides, and $P_{E}$ is the probability that a packet is received erroneously. To model the difference in packet success probability of RTS/CTS and data packets, the time $T_{\mathrm{RCD}}$ is added to the analysis. $T_{\mathrm{RCD}}$ corresponds to the time where the RTS/CTS transmissions succeed whereas the data packet transmission fails, which is given by

$$
T_{\mathrm{RCD}}=T_{\mathrm{RTS}}+T_{\mathrm{CTS}}+T_{\mathrm{data}}+2 T_{\mathrm{SIFS}} \text {, }
$$

where $T_{\mathrm{RCD}}$ is the average time interval of a successful RTS/CTS transmission, but data transmission fails due to channel error. $T_{\mathrm{RTS}}, T_{\mathrm{CTS}}$, and $T_{\text {data }}$ denote the time to transmit a RTS, CTS, and data packet, respectively; $T_{\text {SIFS }}$ represents the duration of SIFS time. The average length of
DIT and throughput based on collision and channel error are, respectively, expressed as

$$
\begin{aligned}
& E\left[l_{\mathrm{DIT}}\right] \\
& =P_{\mathrm{tr}} P_{\mathrm{C}} T_{\mathrm{C}}+P_{\mathrm{tr}} P_{S} P_{\mathrm{PE}}^{\mathrm{RTS}} T_{E}+P_{\mathrm{tr}} P_{S}\left(1-P_{\mathrm{PE}}^{\mathrm{RTS}}\right) P_{\mathrm{PE}}^{\mathrm{CTS}} T_{E} \\
& \quad+P_{\mathrm{tr}} P_{S}\left(1-P_{\mathrm{PE}}^{\mathrm{RTS}}\right)\left(1-P_{\mathrm{PE}}^{\mathrm{CTS}}\right) P_{\mathrm{PE}}^{\mathrm{data}} T_{\mathrm{RCD}}, \\
& E\left[l_{\mathrm{Th}}\right] \\
& =\left(1-P_{\mathrm{tr}}\right) \sigma+P_{\mathrm{tr}} P_{C} T_{\mathrm{C}} \\
& \quad+P_{\mathrm{tr}} P_{S}\left(1-P_{\mathrm{PE}}^{\mathrm{RTS}}\right)\left(1-P_{\mathrm{PE}}^{\mathrm{CTS}}\right) T_{S}+P_{\mathrm{tr}} P_{S} P_{\mathrm{PE}}^{\mathrm{RTS}} T_{E} \\
& \quad+P_{\mathrm{tr}} P_{S}\left(1-P_{\mathrm{PE}}^{\mathrm{RTS}}\right) P_{\mathrm{PE}}^{\mathrm{CTS}} T_{E} \\
& \quad+P_{\mathrm{tr}} P_{S}\left(1-P_{\mathrm{PE}}^{\mathrm{RTS}}\right)\left(1-P_{\mathrm{PE}}^{\mathrm{CTS}}\right)\left(1-P_{\mathrm{PE}}^{\mathrm{data}}\right) P_{\mathrm{PE}}^{\mathrm{ACK}} T_{S},
\end{aligned}
$$

where $\sigma$ is the duration of an empty slot, $P_{\mathrm{PE}}^{\mathrm{CTS}}$ and $P_{\mathrm{PE}}^{\mathrm{ACK}}$ are, respectively, the PER of the CTS and ACK packets, $T_{C}$ and $T_{E}$ are, respectively, the collision and error transmission times (which are composed of the expected transmission time of RTS and CTS packets), and $T_{S}$ is the transmission time in DCF $[6,7]$. When a transmitted packet is not acknowledged properly, retries can be performed up to seven times [1]. Since the probability that a transmitted packet is not acknowledged depends on $p$ and since the backoff time is uniformly chosen in the range $(0, C W-1]$, the DIT of the PS scheme can be derived as in (9). In addition, considering the average length of throughput and the payload length, $L_{\text {data }}$, the throughput of PS scheme is given in (10):

$$
\begin{aligned}
T_{\mathrm{DIT}} & =E\left[l_{\mathrm{DIT}}\right] \sum_{i=0}^{m}\left(\frac{\mathrm{CW}_{i}+1}{2} \frac{p^{i}-p^{m+1}}{1-p^{m+1}}\right), \\
S_{\mathrm{PS}} & =\frac{P_{\mathrm{tr}} P_{S} L_{\mathrm{data}}}{E\left[l_{\mathrm{Th}}\right]} .
\end{aligned}
$$

In the proposed scheme, a new $\mathrm{HO}$ criterion is used which can effectively determine when RTS/CTS packets succeed 


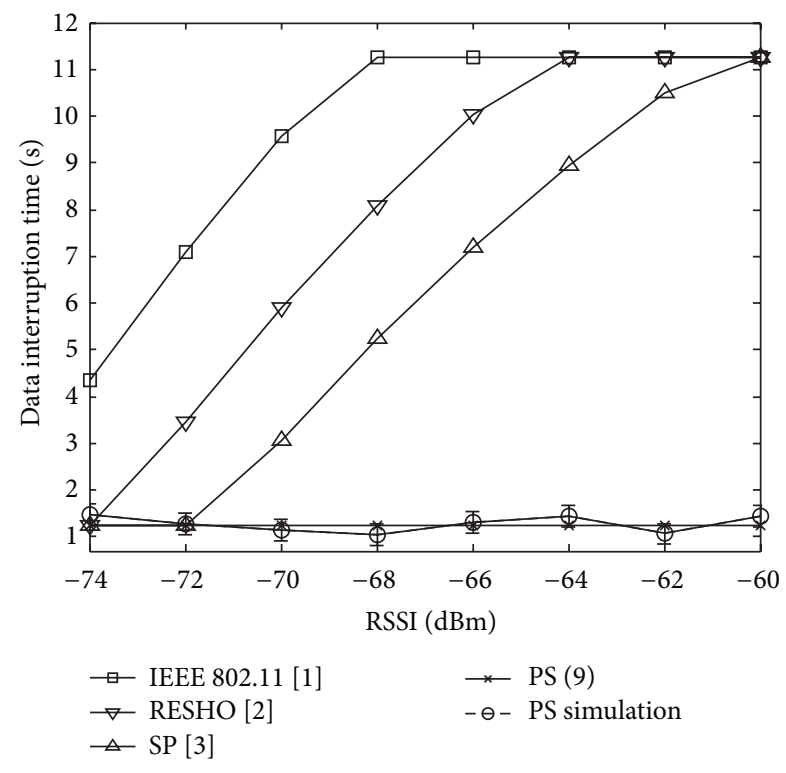

FIgURE 3: Data interruption time versus outage RSSI.

whereas the data packet (or padded Probe Request packet) fails. In addition, a SINR criterion is used to prevent frequent $\mathrm{HO}$ triggering. For the $\mathrm{HO}$ situation where the RSSI is high and the SINR of the data packet is high, the existing schemes of [1-3] may work well. However, in the HO situation where the RSSI is high but the SINR is low such that communication of the data packet consistently fails, the existing schemes of [1-3] do not conduct $\mathrm{HO}$, even though data packet cannot be received, where this decision can cause long DIT. In addition, the PS scheme can avoid the situation where communication of RTS/CTS packets succeeds whereas data packets fail due to low SINR. Due to this reason, the throughput of WLAN networks is improved.

Interference can occur due to various causes (e.g., Bluetooth devices, ZigBee devices, and other Wi-Fi APs and devices using cochannel frequencies), which results in influencing the bit error rate (BER) level. To test the performance of the proposed scheme and compare it to other schemes in poor channel conditions, simulation testing was conducted at relatively high BER levels, which could result in outage based on the HO RSSI threshold of [1-3]. The resource-efficient soft handoff mobility (RESHO) scheme performs HO before the RSSI threshold is passed based on using the speed and direction of the device [2]. The soft proactive (SP) scheme performs proactive session setup by considering the signal strength of the target cell and the mobility of the device [3]. To implement outage circumstances, the BER was set to $3 \times 10^{-4}$ based on the channel model of [4]; in addition, $P_{\mathrm{PE}}^{\mathrm{RTS}}=0.1$ and $P_{\mathrm{PE}}^{\mathrm{data}}=0.95$ were set based on the WLAN simulation model of $[8,9]$.

In addition, the random way point (RWP) mobility model $[10,11]$ was used based on a uniform velocity distribution characterized by $(a, b)=(0.5,2.5) \mathrm{m} / \mathrm{s}$, which models the velocity of walking users. In Figure 3, the UE was designed to maintain connectivity to the WLAN AP until the HO RSSI thresholds based on IEEE 802.11 [1], RESHO [2], SP [3],

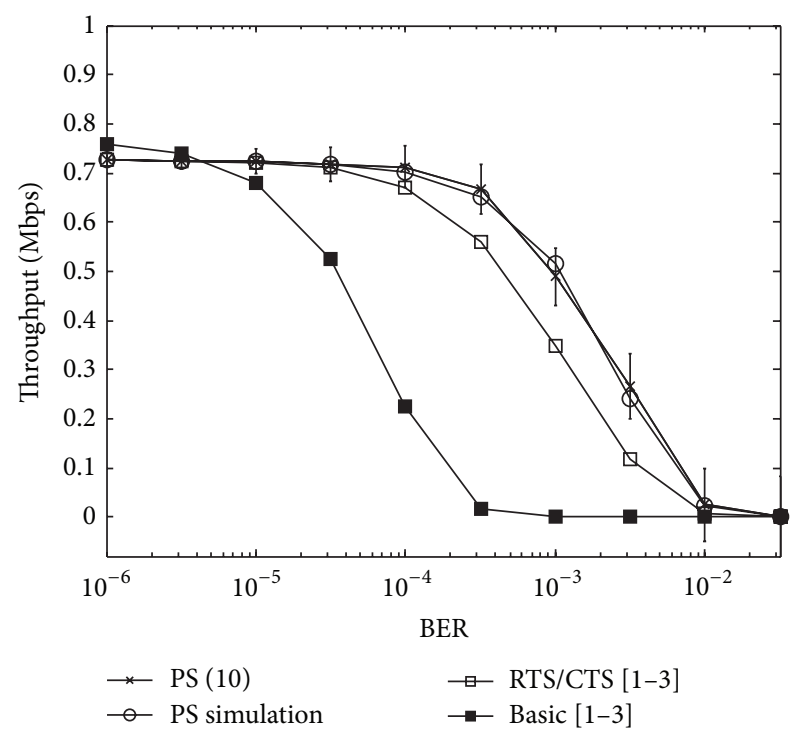

FIGURE 4: Throughput results versus various BER.

and the proposed PS scheme (based on (9)) were exceeded in the simulators programmed using MATLAB [12]. In addition, Network Simulator 2 (NS2) [13] was used to evaluate the DIT performance of the proposed PS scheme to confirm the MATLAB simulation results of (9). In the NS2 simulation, 10,000 iterations were performed to compute the average DIT. For the simulation of the proposed scheme, IEEE 802.11 information of the UE is required. The IEEE 802.11 simulation parameters were based on $[6,14]$ such that the simulation environment is uniform for all performance evaluation models in Figures 3 and 4. The length of the PHY and MAC header was 128 bits and 272 bits, respectively, the data payload was set to 1,450 bytes, and the RTS, CTS, and ACK packets were set to 44 bytes, $n$ was set to 20 , the minimum and maximum contention window sizes were set to 15 and 1,023 slot times, respectively, and the maximum backoff counter was set to 7 . The duration of SIFS and DIFS was set to $10 \mu \mathrm{s}$ and $20 \mu \mathrm{s}$, respectively, and the transmission data rate was $1 \mathrm{Mbps}$ (IEEE $802.11 b)$. The path loss model is based on $[15,16]$, where the power of the received signal at the UE which is $d$ meters away from the AP is $P_{r}(d)=P_{t}-K-10 u \log \left(d / d_{0}\right)+\chi(\mu, \sigma)$, the transmission power was set to $P_{t}=100 \mathrm{~mW}$, the largescale path loss parameters were set as $K=28.7 \mathrm{~dB}$ and $u=4$, the standard deviation of the Gaussian shadowing was set to $\sigma=6 \mathrm{~dB}$, and the correlation distance is $d_{0}=$ $5 \mathrm{~m}$. As shown in (4), the PS scheme uses events of packet success and SINR which are linked closely with the data packets (or padded the Probe Request packets) transmission. In Figure 3, the PS scheme has a constant DIT without being affected by the interference and noise of the environment. The newly derived (9) shows an accurate match when compared to the simulation results in Figure 3. The results show that RESHO has a maximum difference of $9.8 \mathrm{~s}$ compared to the PS scheme when outage occurs at $-64 \mathrm{dBm}$ of RSSI. In the case of SP, even when it performs proactive HO, SP results in a maximum difference of $9.8 \mathrm{~s}$ compared to the PS scheme when outage occurs at the RSSI level of $-60 \mathrm{dBm}$. 
In Figure 4, MATLAB simulation was used to evaluate the throughput performance of the IEEE 802.11 basic mode (which does not use RTS/CTS packets) [1-3], RTS/CTS mode $[1-3]$, and the proposed PS scheme (based on (10)). In addition, NS2 simulation was used to evaluate the throughput performance of the proposed PS scheme to confirm the MATLAB simulation results of (10). In the NS2 simulations, 10,000 iterations were performed to compute the average throughput. In the RTS/CTS mode [1-3], RTS/CTS and data packets are continuously sent over the WLAN as long as the UE's RSSI is above the HO RSSI threshold. In situations where the RSSI is above the HO RSSI threshold whereas the SINR is not sufficient for data packet transmission, $\mathrm{HO}$ will not occur, but data packet transmissions will continuously fail, resulting in serious performance degradation. The proposed PS scheme avoids this degradation as it checks if the WLAN channel condition is suitable for data packet transmission in addition to the RSSI level in making a decision of $\mathrm{HO}$, and, as a result, the proposed PS scheme eliminates the problems that occur in the PPS region, as shown in Figure 4. In Figure 4, a maximum performance gain of $32 \%$ and $97 \%$ can be obtained at the $3 \times 10^{-3}$ BER level by using the PS scheme when compared to using the existing RTS/CTS mode and existing basic mode, respectively.

\section{Conclusion}

Since RSSI is the added value of signal power as well as surrounding noise and interference, channel estimations based on the RSSI can result in poor HO point decisions that result in long DIT when the noise and/or interference is severe. In this paper, the proposed PS scheme determines the HO point based on the PER of data packets and the SINR. By using the PS scheme, WLAN HO with short DIT and an improved throughput performance can be obtained even when the UE is in the AP's PPS range.

\section{Competing Interests}

The authors declare that they have no competing interests.

\section{Acknowledgments}

This research was supported by Basic Science Research Program through the National Research Foundation (NRF) funded by the Ministry of Education, Science and Technology (no. 2013R1A1A2012082) and the Ministry of Science, ICT and Future Planning (MSIP), Korea, under the Information Technology Research Center (ITRC) support program (IITP2016-H8501-16-1007) supervised by the Institute for Information \& Communications Technology Promotion (IITP).

\section{References}

[1] IEEE, "Part 11: wireless LAN Medium Access Control (MAC) and Physical Layer (PHY) specifications," IEEE Std 802.11-2012, 2012.
[2] R. B. Ali and S. Pierre, "On the impact of soft vertical handoff on optimal voice admission control in PCF-based WLANs loosely coupled to 3G networks," IEEE Transactions on Wireless Communications, vol. 8, no. 3, pp. 1356-1365, 2009.

[3] D. Lee, D. Won, M. J. Piran, and D. Y. Suh, "Reducing handover delays for seamless multimedia service in IEEE 802.11 networks," Electronics Letters, vol. 50, no. 15, pp. 1100-1102, 2014.

[4] T. Ali and M. Saquib, "Performance evaluation of WLAN/cellular media access for mobile voice users under random mobility models," IEEE Transactions on Wireless Communications, vol. 10, no. 10, pp. 3241-3255, 2011.

[5] K. Yang, I. Gondal, and B. Qiu, "Multi-dimensional adaptive SINR based vertical handoff for heterogeneous wireless networks," IEEE Communications Letters, vol. 12, no. 6, pp. 438$440,2008$.

[6] I. Tinnirello, G. Bianchi, and Y. Xiao, "Refinements on IEEE 802.11 distributed coordination function modeling approaches," IEEE Transactions on Vehicular Technology, vol. 59, no. 3, pp. 1055-1067, 2010.

[7] M. Laddomada and F. Mesiti, "On the optimization of the IEEE 802.11 DCF: a cross-layer perspective," International Journal of Digital Multimedia Broadcasting, vol. 2010, Article ID 397824, 13 pages, 2010.

[8] Y. Song, X. Zhu, Y. Fang, and H. Zhang, "Threshold optimization for rate adaptation algorithms in IEEE 802.11 WLANs," IEEE Transactions on Wireless Communications, vol. 9, no. 1, pp. 318-327, 2010.

[9] Z. Yuan, H. Venkataraman, and G.-M. Muntean, "MBE: modelbased available bandwidth estimation for IEEE 802.11 data communications," IEEE Transactions on Vehicular Technology, vol. 61, no. 5, pp. 2158-2171, 2012.

[10] C. Bettstetter, H. Hartenstein, and X. Pérez-Costa, "Stochastic properties of the random waypoint mobility model," Wireless Networks, vol. 10, no. 5, pp. 555-567, 2004.

[11] T. Camp, J. Boleng, and V. Davies, "A survey of mobility models for ad-hoc network research," Wireless Communications and Mobile Computing, vol. 2, no. 5, pp. 483-502, 2002.

[12] The MATLAB Simulator, http://www.mathworks.com/products/matlab/.

[13] The ns-3 network simulator, http://www.nsnam.org/.

[14] P. Dong, J. Wang, J. Huang, H. Wang, and Y. Pan, "Improving performance of QoS applications for wireless networks," International Journal of Distributed Sensor Networks, vol. 2015, Article ID 385935, 12 pages, 2015.

[15] T. S. Rappaport, Wireless Communications: Principles and Practice, Prentice Hall, New York, NY, USA, 2002.

[16] T. Ali, M. Saquib, and C. Sengupta, "Vertical handover analysis for voice over WLAN/cellular network," in Proceedings of the IEEE International Conference on Communications (ICC '10), pp. 1-5, Cape Town, South Africa, May 2010. 

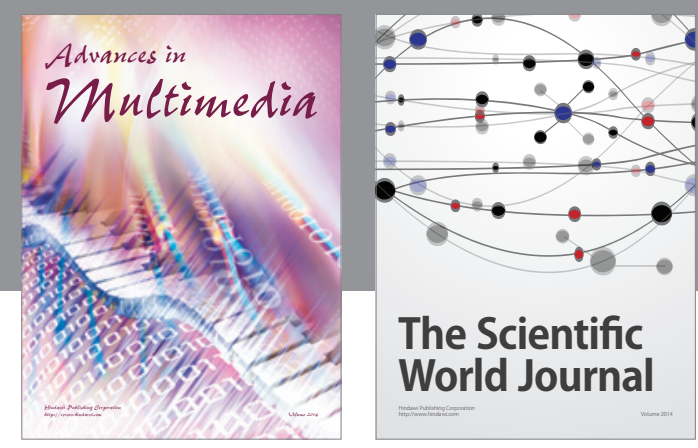

The Scientific World Journal
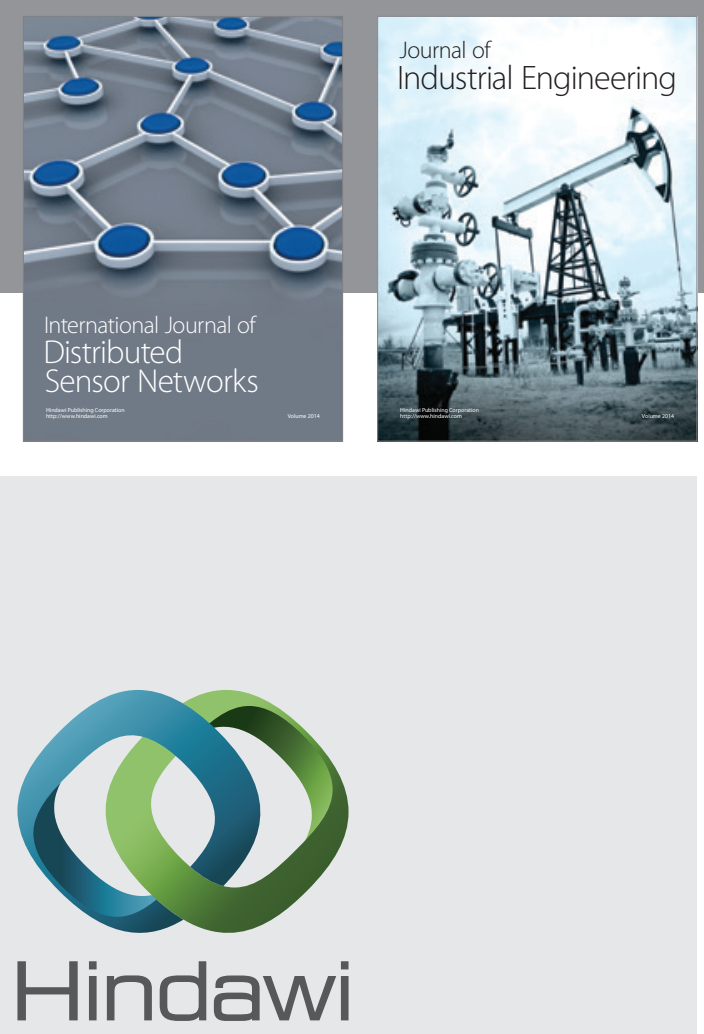

Submit your manuscripts at

http://www.hindawi.com

\section{Computer Networks} and Communications
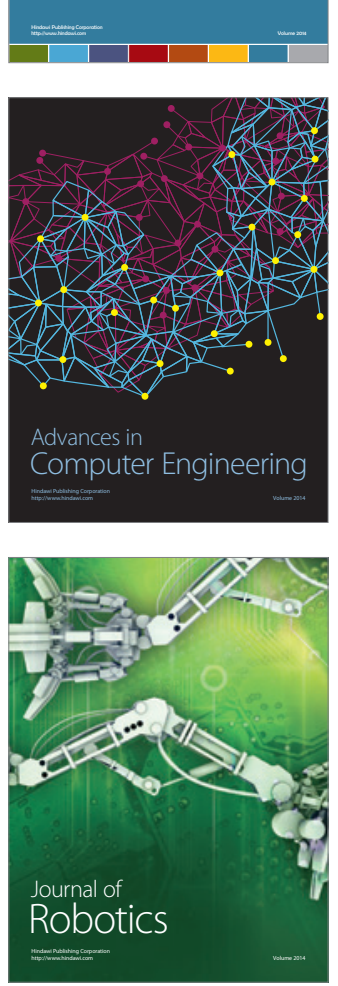
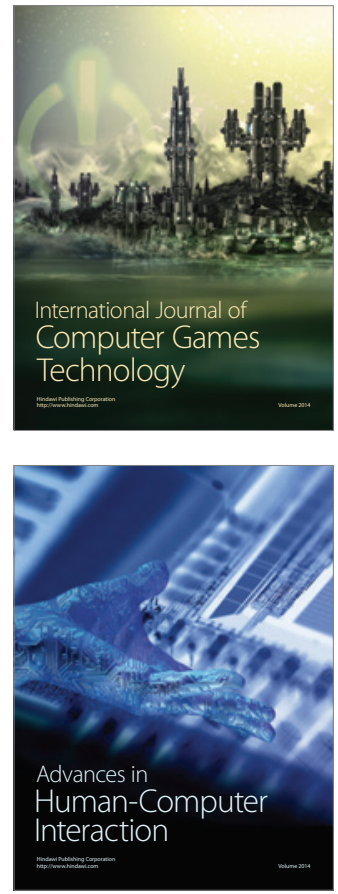
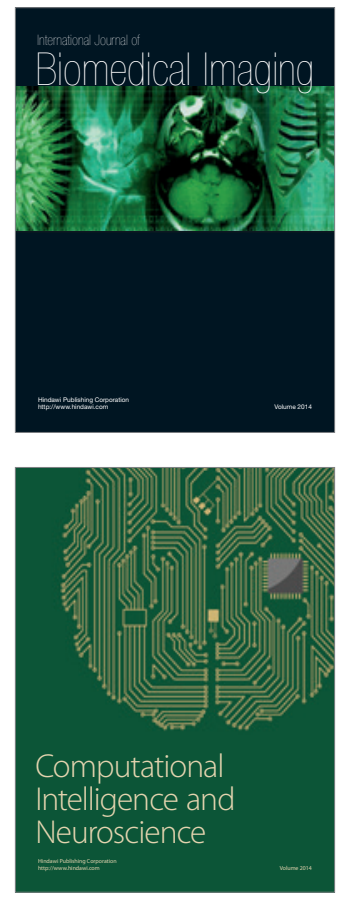
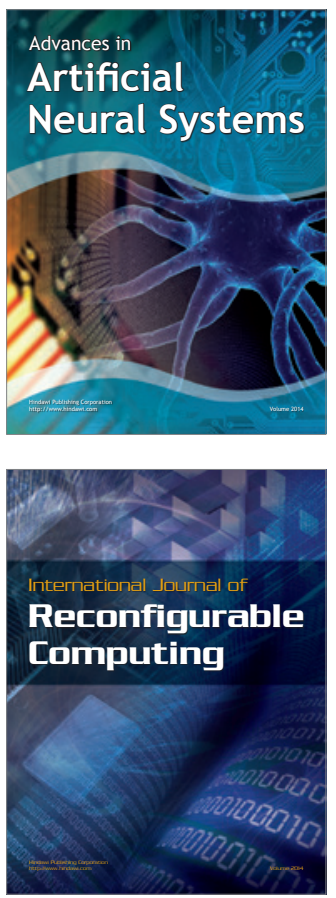
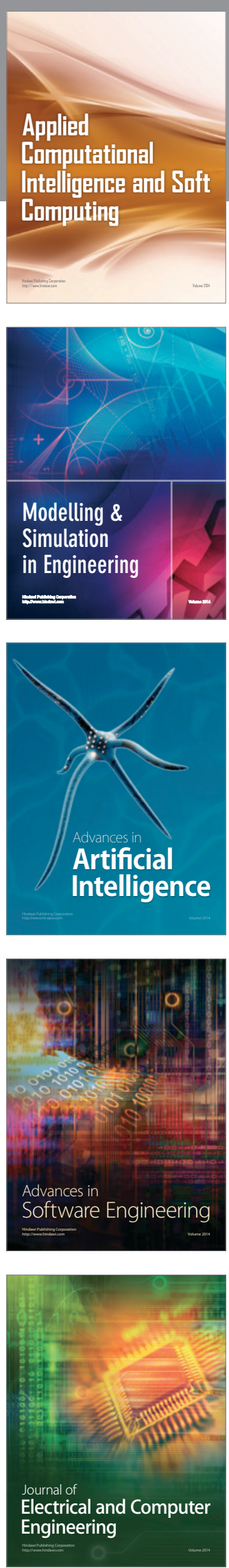